\title{
Effect of thermocycling and varying polymerization techniques on the restorative interface of class $V$ cavities restored with different composite resin systems
}

\author{
Jefferson-Ricardo Pereira ${ }^{1}$, Lindomar-Corrêa Júnior ${ }^{2}$, Marcus-Vinicius-Reis Só ${ }^{3}$, Newton-Fahl Júnior ${ }^{4}$ \\ ${ }^{1}$ DDS, MSc, PhD, Department of Prosthodontics, University of Southern Santa Catarina, Tubarão, Santa Catarina, Brazil \\ ${ }^{2}$ DDS, Department of Prosthodontics, University of Southern Santa Catarina, Tubarão, Santa Catarina, Brazil \\ ${ }^{3}$ DDS, MSc, PhD, Department of Conservative Dentistry, Rio Grande do Sul Federal University, Porto Alegre, RS, Brazil \\ ${ }^{4}$ DDS, MSc, Newton Fahl Center, Curitiba, PR, Brazil
}

Correspondence:

University of Southern Santa Catarina - Tubarão/SC - Brazil

Address: Rua Recife, 200 - Apto 601 - Bairro: Recife

Zip Code: 88701-420

jeffripe@rocketmail.com

Pereira JR, Júnior LC, Só MVR, Júnior NF. Effect of thermocycling and varying polymerization techniques on the restorative interface of class $\mathrm{V}$ cavities restored with different composite resin systems. J Clin Exp Dent. 2017;9(3):e405-9.

http://www.medicinaoral.com/odo/volumenes/v9i3/jcedv9i3p405.pdf

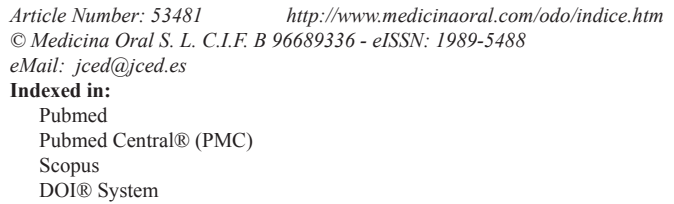

\begin{abstract}
Background: To evaluate marginal microleakage of two composite resins - a methacrylate- and a silorane-based submitted to different polymerization techniques and thermocycling.

Material and Methods: Ninety-six class V cavities were prepared in sound human molars and restored under different polymerization and thermocycling regimens. The adhesive systems employed were Adper Scotchbond Multipurpose and Filtek P-90 for cavities restored with Z250 and P-90. The specimens were restored with Z250 or P-90, and divided into 3 subgroups with different polymerization techniques. The data were analyzed by Three way Analysis of Variance Test $(p<0.05)$.

Results: Micro infiltration lower scores were found in groups which were used silorane-based resin with significant statistical difference compared with the specimens restored with methacrylate-based resin, independently of polymerization type used and thermocycling $(p>0.001)$.

Conclusions: Silorane-based composite resins present lower marginal microleakage values when compared to methacrylate-based composites resins.
\end{abstract}

Key words: Composite resin, microleakage, polymerization.

\section{Introduction}

Dental composites were developed as an esthetic alternative to the long-used amalgam-based restorative materials and have become more popular and widely used in current clinical dentistry $(1,2)$. Benefits incurring from the use of composite resins in lieu of amalgam include the mimicry of natural tooth color, absence of mercury, low thermal conductibility, and the ability to bond to dental structures through adhesive systems (3).

Polymerization is the chemical reaction that converts small molecules, the monomers, into large chains called polymers (4). Light intensity and exposure time have 
been shown to be key factors in determining the speed at which free radicals are released to initiate the polymerization reaction and the extent of cure of composite resins, respectively (5). In this sense, manufacturers have recommended high intensity lights to process a higher conversion degree of monomers into polymers, thus improving the mechanical properties of composite resins. However, conversion degree is always associated with an inconvenience of great clinical relevance: polymerization shrinkage $(6,7)$.

The complete polymerization shrinkage can be divided in pre and post-gel phases. During pre-gel polymerization, flows and tensions inside of material structure are released; during the post-gel phase, however, there is significant stress induction at tooth/restoration interface (8). Because of this, several authors have suggested methods of polymerization that delay pre-gel time, in order to have a slower rate of monomer conversion, which, in turn, allows for a better resin flow with decreased contraction stress. Unlike conventional polymerization, whereby the light intensity is fixed, an initial photoactivation of low light intensity followed by high light intensity final photoactivation, would tend to minimize marginal cracks, without compromising the physical and mechanical properties of composite resins. This process is termed Soft-Start polymerization (9).

Shrinkage generates important clinical drawback, such as gaps between tooth and restoration $(10,11)$. The occurrence of these gaps is due to the forces generated in the material body, which are transmitted to the tooth/ restoration interface. It compromises the bond strength and marginal integrity. The restoration becomes consequently more susceptible to infiltration, secondary caries and postoperative sensitivity (12).

Recent research in the field of dental materials introduced silorane-based composite resins in substitution of methacrylate-based composite resins (13). This material is derivative of oxirane and siloxane molecules, which polymerize themselves with an opening of cationic ring, in maximum overcoming to the clinical inconvenience of material polymerization shrinkage related to methacrylate-based composite resins (14). Furthermore, this material has shown good mechanical properties (15) and fluid stability in oral cavities simulators (16), which encourages its clinical use.

In light of the above, the objective of this study was to evaluate the effect of thermocycling and of varying polymerization techniques on the restored surfaces of Class $\mathrm{V}$ cavities with different composite resins. The hypothesis of this study was that there would be significant differences between the composite resins, and among the intensity polymerization, and thermocycling variables.

\section{Material and Methods}

This study was approved by Unisul ethics comitte. For- ty-eight sound human molars were selected from a Teeth Bank of the University of Southern Santa Catarina. The teeth had been recently extracted by periodontal or orthodontic indications and stored in 10\% formalin solution, in order to maintain their integrity (17).

Teeth were cleaned with water-pumice slurry in a prophylaxis rubber cup and rinsed with copious water before preparation of the cavities. All the teeth were carefully observed through a $4 \mathrm{X}$ binocular loupe (Bio Art Equipamentos Odontológicos Ltda, São Carlos-SP, Brazil) in order to appraise the absence of cracks, deterioration, fissures, or previous restorations, which could compromise final results.

Class V cavities were prepared on the buccal and lingual aspects of all teeth. The cavities dimensions were $3 \mathrm{~mm}$ mesio-distally, $3 \mathrm{~mm}$ cervico-occlusally and $3 \mathrm{~mm}$ deep. The cavities were performed using a cylindrical diamond bur with rounded end (\#2143, KG Sorensen, São Paulo, SP, Brazil) at high speed and under air/water cooling with a cursor that limits the drill action to a predetermined depth. Finishing of the cavity preparations was carried out also under water cooling using fine-gritted diamond burs of the same diameter, (\#2143 F - KG Sorensen, São Paulo, SP, Brazil). The dimensions of the cavities were verified using a precision digital caliper (Digimatic, 500-181U; Mitutoyo Corp, Tokio, Japan). Between each stage of this experiment, teeth were stored in $37^{\circ} \mathrm{C}$ distilled water. All 48 teeth were randomly divided in 12 experimental groups. Six groups were restored using a methacrylate-based resin and a total etch three-step adhesive system ( $\mathrm{Z}$ ) and six of them using a silorane-based restorative material and a self-etch onestep adhesive system developed for silorane-based restorative system $(\mathrm{P})$, these groups were divided into 3 subgroups and subsequently subjected to high $(\mathrm{H})$, softstart (S), and low intensity polymerization (L). Considering similar groups, one was submitted to thermocycling (T) while the other to no thermocycling (N) (Table 1). For the groups restored with Z250, after prepare the cavity, the cavities were etched during 15 seconds and it was washed with air spray and water during de 10 seconds. Humidity excess was dried with cotton pellet without air drying. The Scotchbond Multipurpose primer was applied with a microbrush, the excess was suctioned with an endodontic suction tip, and, after 5 minutes, the Scotchbond Multipurpose bond agent was applied with a microbrush in only one layer, the excess was suctioned with an endodontic suction tip and light-cured for 10 seconds with a curing unit (Blue Fase, Ivoclar Vivadent) using a 9mm-diameter light guide and keeping a distance between the light guide surface and the specimens near $1 \mathrm{~mm}$.

For the groups restored with P-90 the dentin was not etched. The methodology to use silorane adhesive system was exactly the same using scothbond system. 
Table 1. The groups presented in this study. All of material were from 3M ESPE, USA.

\begin{tabular}{|c|c|c|c|c|}
\hline Group & Adhesive system & Composite resin & Polymerization & Thermocycling \\
\hline ZLN & Adper Scotchbond Multi-Purpose & Z250 & Low & No \\
\hline ZLT & Adper Scotchbond Multi-Purpose & Z250 & Low & Yes \\
\hline ZSN & Adper Scotchbond Multi-Purpose & Z250 & Soft-Start & No \\
\hline ZST & Adper Scotchbond Multi-Purpose & Z250 & Soft-Start & Yes \\
\hline ZHN & Adper Scotchbond Multi-Purpose & Z250 & High & Yes \\
\hline ZHT & Adper Scotchbond Multi-Purpose & Z250 & High & No \\
\hline PLN & Filtek P-90 & P-90 & Low & No \\
\hline PLT & Filtek P-90 & P-90 & Soft-Start & Yes \\
\hline PSN & Filtek P-90 & P-90 & Soft-Start & No \\
\hline PST & Filtek P-90 & P-90 & High & Yes \\
\hline PHN & Filtek P-90 & Piltek P-90 & & \\
\hline PHT & & & & \\
\hline
\end{tabular}

Composite resins were applied in the different groups using the incremental technique by insertion of three increments: two of them oblique and one covering both previous increments. In the groups ZLN, PLN, ZLT and PLT, the photo activation of each increment was performed by the technique Low with fixed light intensity at $650 \mathrm{~mW} / \mathrm{cm}^{2}$ for $20 \mathrm{~s}$; in the groups ZSN, PSN, ZST and PST, the photo activation in each increment was performed by Soft-Start technique at starting in $0 \mathrm{~mW} / \mathrm{cm}^{2}$ to $650 \mathrm{~mW} / \mathrm{cm}^{2}$ in $5 \mathrm{~s}+1200 \mathrm{~mW} / \mathrm{cm}^{2}$ for $20 \mathrm{~s}$; in the groups ZHN, PHN, ZHT and PHT, the photo activation in each increment was performed by the technique High in $1200 \mathrm{~mW} / \mathrm{cm}^{2}$. All of them using the curing unit Blue Fase (Ivoclar-Vivadent). Restorations were finalized using finishing burs (KG Sorensen, São Paulo, SP, Brazil) in order to remove gross excess, followed by sof-lex disc (3M ESPE).

After finishing the specimens from groups ZLT, PLT, ZST, PST, ZHT, and PHT, they were taken to the thermocycling and submitted to 5000 cycles during 30 seconds in $5^{\circ}$ and $55^{\circ} \mathrm{C}$ temperature, while specimens from groups ZLN, PLN, ZSN, PSN, ZHN, and PHN stayed immersed in $37^{\circ} \mathrm{C}$ distilled water.

Specimens were embedded in $1 \%$ methylene blue (Prolabo, Paris, France) during 48 hours. The evaluation of pigment penetration in the interfaces was performed after specimens were washed in distilled water. Microleakage analysis was performed under binocular microscope (X10, model S2H, Olympus corp., Tokyo, Japan) after each crown was cross-sectioned buccolingually using a diamond saw (Isomet 1000, Buehler, Lake Bluff, Ill, USA) at $400 \mathrm{rpm}$ and $250 \mathrm{~g}$, under copious watercooling.
Evaluation criteria consisted in scores from 0 to 4 which mean: score 0 - absence of infiltration; 1 - Infiltration until the half of surrounding wall; 2- Infiltration in all surrounding wall; 3 - Infiltration in surrounding wall and axial; 4- Infiltration in surrounding wall and axial towards the pulp. The results founded were statistically analyzed by Three way Analysis of Variance Test $(p<0.05)$.

\section{Results}

Table 2 shows microleakage scores found in different groups, which were combined composite resins materials, polymerization systems, and thermocycling. Table

Table 2. Microleakage scores found in different groups which were combined composite resins materials, polymerization systems, and thermocycling.

\begin{tabular}{|l|c|c|c|}
\hline Group & Mean & N & Std. Dev. \\
\hline ZLN & 1,312500 & 16 & 1,138347 \\
\hline PLN & 0,625000 & 16 & 0,718795 \\
\hline ZSN & 1,437500 & 16 & 1,459166 \\
\hline PSN & 0,437500 & 16 & 0,512348 \\
\hline ZHN & 2,000000 & 16 & 1,505545 \\
\hline PHN & 0,812500 & 16 & 0,834166 \\
\hline ZLT & 1,437500 & 16 & 0,963933 \\
\hline PLT & 0,812500 & 16 & 0,910586 \\
\hline ZST & 1,750000 & 16 & 1,064581 \\
\hline PST & 0,687500 & 16 & 0,873212 \\
\hline ZHT & 1,750000 & 16 & 0,856349 \\
\hline PHT & 1,125000 & 16 & 1,024695 \\
\hline
\end{tabular}


3 shows microleakage scores found considering separately composite resins materials, polymerization systems, and thermocycling.

It was possible verify that micro infiltration lower scores were found in groups which were used silorane-based resin with significant statistical difference compared with the specimens restored with methacrylate-based resin, independently of polymerization type used and thermocycling $(p>0.001)$. For other groups there were no statistical significant differences among them.

Microleakage scores were submitted to statistical analysis by three way Analysis of Variance Test in order to verify the existence of difference among tested groups (Table 4).

Table 3. Microleakage scores found in different groups considering separately composite resins materials, polymerization systems, and thermocycling.

\begin{tabular}{|l|c|c|}
\hline Group & Mean & N \\
\hline Z-250 & 1.615 & 86 \\
\hline P-90 & 0.75 & 86 \\
\hline Low & 1.047 & 64 \\
\hline Soft & 1.078 & 64 \\
\hline High & 1.422 & 64 \\
\hline No thermo & 1.104 & 86 \\
\hline Thermo & 1.260 & 86 \\
\hline All Grps & 1,182292 & 192 \\
\hline
\end{tabular}

\section{Discussion}

The results of this study accepted the hypothesis that there were significant differences between the composite resins systems, polymerization system, and thermocycling. It was possible to observe that significant lower microleakage scores were found in groups restored with silorane based resin and it was no found significant differences comparing the different groups according in thermocycling and polymerization. This fact can be explained by polymerization kinetics of silorane that can be slower and show the property of cationic ring opening able to reduce its contraction to levels lower than $1 \%$ volume $(14,18)$.

Even thought, Filtek silorane presents a filler load of $53 \%$ by volume whereas Z-250 presents a filler load of $60 \%$. This might provide a better flow of silorane-based materials, which develop lower stress during polymerization and, consequently, better marginal integrity maintenance when compared to methacrylate-based composites. This fact could be observed in this study when comparing the different composite resins.

The results of this study showed that there is no statistical difference among groups with or without thermocycling, even in silorane-based composite resin or methacrylatebased composite resin. These results are in accordance with Eakle (19) who found that there is no difference in microleakage when the samples are submitted or not to the thermal challenge. According to Veronezi et al. (20), the difference in thermal expansion between the material and the dental structure provokes alternating increase and decrease of space between material and

Table 4. Three Way Analysis of Variance.

\begin{tabular}{|c|c|c|c|c|c|}
\hline \multirow{2}{*}{ Source of Variation } & \multirow{2}{*}{ DF } & \multirow{2}{*}{ SS } & \multirow{2}{*}{ MS } & $\mathrm{F}$ & \multirow{2}{*}{$P$} \\
\hline & & & & 34.16 & \\
\hline Composite resin & 1 & 35.880 & 35.888 & 0 & $<0.001$ \\
\hline Photopolimerization & 2 & 5.542 & 2.771 & 2.638 & 0.074 \\
\hline Thermocycling & 1 & 1.172 & 1.172 & 1.116 & 0.292 \\
\hline Composite resin $\mathrm{x}$ Photopolimerization & 2 & 1.167 & 0,583 & 0,555 & 0.575 \\
\hline Composite resin xTermicycling & 1 & 0,422 & 0,422 & 0,402 & 0.527 \\
\hline Photopolimerization $\mathrm{x}$ Thermocycling & 2 & 0,5 & 0,25 & 0,238 & 0.788 \\
\hline \multicolumn{6}{|l|}{ Composite resin $\mathrm{x}$ Photopolimerization $\mathrm{x}$} \\
\hline \multirow[t]{2}{*}{ Thermocycling } & \multirow[t]{2}{*}{2} & 0,875 & \multirow[t]{2}{*}{0,438} & \multirow[t]{2}{*}{0,417} & \multirow[t]{2}{*}{0.66} \\
\hline & & 189.06 & & & \\
\hline \multirow[t]{2}{*}{ Residual } & 180 & 3 & \multirow[t]{2}{*}{1.050} & & \\
\hline & & 234.62 & & & \\
\hline Total & 191 & 0 & 1.228 & & \\
\hline
\end{tabular}


dental structure, which can provide an increase in marginal microleakage in composite resin restoration. The incremental technique may be reduced the contraction and consequently the microleakage of all restoration.

Another important factor that may have influenced the higher microleakage was the high intensity polymerization regimen, as it provides a prolonged duration of light exposure under high intensity. According to some authors, this light activation method is ideal for polymerizing composite resins because it provides adequate polymerization depth with improved physical and mechanical properties (21). On the other hand, some authors assert that this type of polymerization may not prolong pre-gel stage, which is responsible for a great part of compensation of polymerization contraction. In this way, crosslinks are rapidly established and the flow ability of resin and every contraction stress generated is transferred to the bond interface tooth/restoration. It can compromise the marginal integrity and increase microleakage levels (22). These finds could not be observed in the present study and can be explained by the use of the incremental technique.

Despite the influence of thermocycling factors and polymerization methods, this study highlights the chemical composition of material above other factors. Even with the possibility of thermal influence and of current different polymerization methods that prolong the pregel stage, this study did not evidence the influence of these factors in groups tested with either silorane-based or with metacrylate-based composite resins. In these groups there is no statistically significant differences, which allows the inference as more important factor in marginal microleakage decrease being the chemical composition of silorane and its ability of opening in the moment of polymerization.

\section{Conclusions}

Considering the results of this study, it is possible conclude that silorane presents low values of marginal microleakage when compared to methacrylate-based composites. These results seem to be associated to the chemical composition inherent to the material, instead of to different polymerization systems and to the thermal challenge promoted by thermo cycling.

\section{References}

1. Buergers R, Schneider-Brachert W, Hahnel S, Rosentritt M, Handel G. Streptococcal adhesion to novel low-shrink silorane-based restorative. Dent Mater. 2009;25:269-75.

2. Marchesi G, Breschi L, Antoniolli F, Di Lenarda R, Ferracane J, Cadenaro M. Contraction stress of low-shrinkage composite materials assessed with different testing systems. Dent Mater. 2010;26:947-53. 3. Santini A, Vesna M. Comparison of the hybrid layer formed by silorane adhesive, one-step self-etch and etch and rinse systems using confocal micro-Raman spectroscopy and SEM. J Dent. 2008;36:683-91.

4. Anusavice KJ. Phillips science of dental materials. Philadelphia: W.B Saunders Co; 1996.

5. Rueggeberg FA, Caughman WF, Curtis JW. Effect of light inten- sity and exposure duration on cure of resin composite. Oper Dent. 1994; 19:26-32.

6. Plasse NP, Wenger F, Picard B, Colon P. Evaluation of microleakage of composite resin restorations by an electrochemical technique: the impedance methodology. Dent Mater. 2004;20:425-34.

7. Taha NA, Palamara JEA, Messer HH. Cuspal deflection, strain and microleakage of endodontically treated premolar teeth restored with direct resin composites. J Dent. 2009;37:724-30.

8. Chandurkar AM, Metgud SS, Yakub SS, Usumez S. Evaluation of microleakage in class $v$ composite restoration using different techniques of polymerization. Int J Prosthodont Restor Dent. 2012;31:10-5.

9. Chan DCN, Browning WD, Frazier KB, Brackett MG. Clinical evaluation of the soft-start (Pulse-delay) polymerization technique in class I and II composite restorations. Oper Dent. 2008;33:265-71.

10. Alomari Q, Ajlouni R, Omar R. Managing the polymerization shrinkage of resin composite restorations: a review. SABJ. 2007;62:12-8

11. Gerdolle DA, Mortier E, Droz D. Microleakage and polymerization shrinkage of various polymer restorative materials. J Dent Child. 2008; 75:125-33

12. Watts DC, Hindi A. Intrinsic soft start polymerization shrinkage - kinetic in an acrylic based resin composite. Dent Mater. 1999;15:3945 .

13. Pereira JR, Júnior LC, de Souza Almeida M, do Valle AL, Honório HM, Vidotti HA, et al. Effect of silorane-based adhesive system on bond strength between composite and dentin substrate. J Conserv Dent. 2015;18:488-91.

14. Weinmann W, Thalacker C, Guggenberger R. Siloranes in dental composites. Dent Mater. 2005;21:68-74.

15. Ilie N, Kunzelmann KH, Hickel R. Evaluation of micro-tensile bond strengths of composite materials in comparison to their polymerization shrinkage. Dent Mater. 2006;22:593-601.

16. Eick DJ, Smith ER, Pinzino SC, Kostoryz EL. Stability of silorane dental monomers in aqueous systems. J Dent. 2006;34:405-10.

17. Ferrari M, Goracci C, Sadek F, Kostoryz EL. Microtensile bond strength tests: scanning electron microscopy evaluation of sample integrity before testing. Eur J Oral Sci. 2002;110:385-91.

18. Eakle WS. Effect of thermal cycling on fracture strength and microleakage in teeth restored with a bonded composite resin. Dent Mater. 1986;2:114-7.

19. Soares GP, Ambrosano GM, Lima DA, Marchi GM, CorrerSobrinho L, Lovadino JR, et al. Effect of light polymerization time, mode, and termal and mechanical load cycling on microleakage in resin composite restorations. Lasers Med Sci. 2014;29:545-50.

20. Mehl A, Hickel R, Kunzelmann KH. Physical properties and gap formation of light-cured composites with and without "softstart-polymerization". J Dent. 1997;25:321-30.

21. Mehl A, Hickel R, Kunzelmann KH. Physical properties and gap formation of light-cured composites with and without "softstart-polymerization". J Dent 1997; 25:321-30.

22. Lopes MB, Serralvo AD, Felizardo KR, et al. Evaluation of the flexural resistance and stress contraction of a silorane-based composite submitted to different protocols of polymerization. Appl Adhes Sci. 2014;2:23.

Conflict of Interest

The authors have declared that no conflict of interest exist. 